\title{
Asymptotic Properties of Generalized Eigenfunctions for Three Body Schrödinger Operators
}

\section{Hiroshi Isozaki}

Department of Mathematics, Osaka University, Toyonaka, 560, Japan

Received February 21, 1992

Abstract. We study the spatial asymptotics of generalized eigenfunctions of three body Schrödinger operators and derive all the S-matrices with initial state of 2 clusters.

\section{Introduction}

This paper is a continuation of our previous work [2] and deals with properties of S-matrices for three body Schrödinger operators. We first recall the basic notation and results of [2]. In $\mathbf{R}^{3}$ we consider three particles with mass $m_{i}$ and position $x^{i}$. Let $\alpha$ be a pair $(i, j)$ and

$$
\begin{gathered}
x^{\alpha}=\sqrt{2 m_{\alpha}}\left(x^{i}-x^{j}\right), \quad x_{\alpha}=\sqrt{2 n_{\alpha}}\left(x^{k}-\frac{m_{i} x^{i}+m_{j} x^{j}}{m_{i}+m_{j}}\right), \\
\frac{1}{m_{\alpha}}=\frac{1}{m_{i}}+\frac{1}{m_{j}}, \quad \frac{1}{n_{\alpha}}=\frac{1}{m_{k}}+\frac{1}{m_{i}+m_{j}} .
\end{gathered}
$$

Then the Schrödinger operator is defined by

$$
H=H_{0}+\sum_{\alpha} V_{\alpha}\left(x^{\alpha}\right), \quad H_{0}=-\Delta_{x^{\alpha}}-\Delta_{x_{\alpha}}
$$

on $L^{2}(X)$, where $X=\left\{\left(x^{1}, x^{2}, x^{3}\right) ; \sum_{i=1}^{3} m_{i} x^{i}=0\right\}$. We consider wave operators, known to exist when $V_{\alpha}\left(x^{\alpha}\right)$ 's decay faster than $\left|x^{\alpha}\right|^{-1}$,

$$
\begin{aligned}
& W_{0}^{ \pm}=\mathrm{s}-\lim _{t \rightarrow \pm \infty} e^{i t H} e^{-i t H_{0}}, \\
& W_{\alpha}^{ \pm}=s-\lim e^{i t H} e^{-i t H_{\alpha}} J_{\alpha}, \\
& H_{\alpha}=H_{0}+V_{\alpha}, \quad\left(J_{\alpha} f\right)\left(x^{\alpha}, x_{\alpha}\right)=u_{\alpha}\left(x^{\alpha}\right) f\left(x_{\alpha}\right) \text {, }
\end{aligned}
$$


where $u_{\alpha}$ is a normalized eigenfunction of $h^{\alpha}=-\Delta_{x^{\alpha}}+V_{\alpha}\left(x^{\alpha}\right)$ with eigenvalue $E^{\alpha}<0$. We define the scattering operator $S_{0 \alpha}$ by

$$
S_{0 \alpha}=\left(W_{0}^{+}\right)^{*} W_{\alpha}^{-} .
$$

Let $\mathscr{F}_{0}: L^{2}\left(\mathbf{R}^{6}\right) \rightarrow L^{2}\left((0, \infty) ; L^{2}\left(S^{5}\right)\right)$ and $\mathscr{F}_{\alpha}: L^{2}\left(\mathbf{R}^{3}\right) \rightarrow L^{2}\left(\left(E^{\alpha}, \infty\right) ; L^{2}\left(S^{2}\right)\right)$ be the unitary operators defined by

$$
\begin{gathered}
\left(\mathscr{F}_{0} f\right)(\lambda, \theta)=C_{0}(\lambda) \int_{\mathbf{R}^{6}} e^{-i \sqrt{\lambda} \theta \cdot x} f(x) d x, \\
C_{0}(\lambda)=(2 \pi)^{-3} 2^{-1 / 2} \lambda, \\
\left(\mathscr{F}_{\alpha} f\right)(\lambda, \omega)=C_{\alpha}(\lambda) \int_{\mathbf{R}^{3}} e^{-i \sqrt{\lambda-E^{\alpha}} \omega \cdot x} f(x) d x, \\
C_{\alpha}(\lambda)=(2 \pi)^{-3 / 2} 2^{-1 / 2}\left(\lambda-E^{\alpha}\right)^{1 / 4} .
\end{gathered}
$$

Let

$$
\hat{S}_{0 \alpha}=\mathscr{F}_{0} S_{0 \alpha} \mathscr{F}_{\alpha}^{*} .
$$

Then the S-matrix $\hat{S}_{0 \alpha}(\lambda) \in \mathbf{B}\left(L^{2}\left(S^{2}\right) ; L^{2}\left(S^{5}\right)\right)$ is defined by the relation

$$
\left(\hat{S}_{0 \alpha} f\right)(\lambda, \theta)=\left(\hat{S_{0 \alpha}}(\lambda) f(\lambda, \cdot)\right)(\theta)
$$

for all $\lambda>0, \theta \in S^{5}$ and $f \in L^{2}\left(\left(E^{\alpha}, \infty\right) ; L^{2}\left(S^{2}\right)\right)$. We assume that $V_{\alpha}$ is a real $C^{\infty}$-function such that for a constant $\rho>0$

$$
\left|\partial_{y}^{m} V_{\alpha}(y)\right| \leqq C_{m}(1+|y|)^{-\rho-m}, \quad m=0,1,2, \ldots,
$$

$\partial_{y}^{m}$ denoting an arbitrary derivative of $m^{\text {th }}$ order with respect to $y$. Let

$$
\begin{aligned}
& X_{\beta}=\left\{x \in X ; x^{\beta}=0\right\}, \\
& M=S^{5} \backslash \bigcup_{\beta} X_{\beta}, \quad N=S^{5} \cap\left(\bigcup_{\beta} X_{\beta}\right) .
\end{aligned}
$$

Then in [2] we showed the following results.

(1) If $\rho>4+1 / 2, \hat{S}_{0 \alpha}(\lambda)$ has a continuous kernel outside $N$ :

$$
\hat{S}_{0 \alpha}(\lambda ; \theta, \omega) \in C\left((0, \infty) \times M \times S^{2}\right) .
$$

(2) If $\rho>5+1 / 2$, we have the following asymptotic expansion around $N$ :

$$
\hat{S}_{0 \alpha}(\lambda ; \theta, \omega) \simeq\left|\theta^{\beta}\right|^{-1} A_{\beta,-1}\left(\lambda ; \frac{\theta^{\beta}}{\left|\theta^{\beta}\right|}, \theta_{\beta}, \omega\right)+A_{\beta, 0}\left(\lambda ; \frac{\theta^{\beta}}{\left|\theta^{\beta}\right|}, \theta_{\beta}, \omega\right),
$$

as $\left|\theta^{\beta}\right| \rightarrow 0$, where we decompose $\theta \in S^{5}$ as $\theta=\left(\theta^{\beta}, \theta_{\beta}\right)$ in accordance with the choice of the Jacobi coordinates. The coefficient $A_{\beta,-1}$ can be written explicitly in terms of 2-cluster scattering amplitudes associated with eigenfunctions with zero eigenvalues and the zero-resonance of $h^{\beta}$.

In this paper, we relate this S-matrix to the asymptotic behavior of the generalized eigenfunction for $H$. 
We first recall the 2-body problem. The generalized eigenfunction $\varphi(x, \xi)$ of $-\Delta+V$ in $\mathbf{R}^{n}$ is written as

$$
\begin{aligned}
\varphi(x, \xi) & =e^{i x \xi}-v, \\
v & =v(x, \xi)=\left(-\Delta+V-|\xi|^{2}-i 0\right)^{-1}\left(V(x) e^{i x \xi}\right) .
\end{aligned}
$$

The first term, $e^{i x \xi}$, represents the incident wave, and the second term, $v$, the scattered wave. The scattering amplitude $A(\lambda ; \theta, \omega)=\hat{S}(\lambda ; \theta, \omega)-\delta(\theta-\omega)$ is derived from $v$ in the following way:

$$
\begin{aligned}
v(x, \sqrt{\lambda} \omega) & \sim C(\lambda) r^{-(n-1) / 2} e^{i \sqrt{\lambda} r} A(\lambda ; \theta, \omega), \\
\theta & =x /|x|, \quad r=|x| \rightarrow \infty .
\end{aligned}
$$

In the case of the three body problem, our generalized eigenfunction is given by

$$
\begin{aligned}
\varphi(x, \lambda, \omega) & =e^{i \sqrt{\lambda-E^{\alpha}} \omega \cdot x_{\alpha}} u_{\alpha}\left(x^{\alpha}\right)-v, \\
v & =R(\lambda+i 0) f, \quad R(z)=(H-z)^{-1}, \\
f & =f(x, \lambda, \omega)=\sum_{\gamma \neq \alpha} V_{\gamma}\left(x^{\gamma}\right) u_{\alpha}\left(x^{\alpha}\right) e^{i \sqrt{\lambda-E^{\alpha}} \omega \cdot x_{\alpha}} .
\end{aligned}
$$

The scattering matrix $\hat{S}_{0 \alpha}(\lambda)$ is related to the spatial asymptotics of $v$ in the same way as in the 2-body problem.

Theorem 1.1. If $\rho>4+1 / 2$, for any $\lambda>0$,

$$
\begin{aligned}
\mathrm{s}-\lim _{r \rightarrow \infty} r^{5 / 2} e^{-i \sqrt{\lambda} r} v(r \cdot) & =C_{1}(\lambda) \hat{S_{0 \alpha}}(\lambda ; \cdot, \omega), \\
C_{1}(\lambda) & =e^{-\pi i / 4} 2 \pi \lambda^{-1 / 4}\left(\lambda-E^{\alpha}\right)^{-1 / 4},
\end{aligned}
$$

in $L_{\text {loc }}^{2}(M)$.

However, it is not easy to replace $M$ by $S^{5}$ in the above theorem, since the behavior of $v$ in a neighborhood of $N$ is rather complicated. What we can expect is the limit in an averaged sense.

We take $\chi_{\beta}(x) \in C^{\infty}(X)$ such that $\chi_{\beta}(x)=1$ if $\left|x^{\beta}\right| /|x|<\varepsilon, \chi_{\beta}(x)=0$ if $\left|x^{\beta}\right| /|x|>2 \varepsilon$, and $\rho_{+}(t) \in C^{\infty}\left(\mathbf{R}^{1}\right)$ such that $\rho_{+}(t)=1$ if $t>1-\varepsilon, \rho_{+}(t)=0$ if $t<1-2 \varepsilon$, where $\varepsilon$ is a small positive constant. We also take $\rho(t) \in C_{0}^{\infty}((0, \infty))$ such that $\int_{0}^{\infty} \rho(t) d t=1$. In order to facilitate the proof, we assume that the pair potentials are rapidly decreasing, but the following two theorems can of course be proved for more slowly decreasing potentials.

Theorem 1.2. Suppose that $V_{\alpha}$ 's are rapidly decreasing functions. Then

$$
\begin{gathered}
\mathrm{s}-\lim _{R \rightarrow \infty} \frac{1}{R} \int_{\mathbf{R}^{6}} e^{-i \sqrt{\lambda} \theta \cdot x} \theta_{\beta} \cdot \hat{x}_{\beta} \rho_{+}\left(\frac{\theta_{\beta}}{\left|\theta_{\beta}\right|} \cdot \frac{x_{\beta}}{\left|x_{\beta}\right|}\right) \chi_{\beta}(x) \rho\left(\frac{\left|x_{\beta}\right|}{R}\right) v(x) d x \\
=C_{2}(\lambda) \hat{S_{0 \alpha}}(\lambda ; \theta, \omega), \\
C_{2}(\lambda)=-(2 \pi)^{7 / 2} \lambda^{-3 / 2}\left(\lambda-E^{\alpha}\right)^{-1 / 4},
\end{gathered}
$$

in $L^{2}\left(\tilde{N}_{\beta}\right)$, where $\tilde{N}_{\beta}$ is a small neighborhood of $N \cap X_{\beta}$ and $\hat{x}_{\beta}=x_{\beta} /\left|x_{\beta}\right|$. 
In the neighborhood of the $X_{\beta}$-plane, there are two sorts of scattering, the 3 -cluster scattering and the 2-cluster scattering. We can distinguish between them by changing the way of taking the limit at infinity of $v$.

Let $u_{\beta l}\left(x^{\beta}\right)$ be a normalized eigenfunction of $h^{\beta}$ with eigenvalue $E_{l}^{\beta} \leqq 0$. Let $A_{\beta l}\left(\lambda ; \theta_{\beta}, \omega\right)$ be the 2-cluster scattering amplitude associated with the process in which, after the collision, the pair $\beta$ takes the bound state $u_{\beta l}$.

Theorem 1.3. Suppose that $V_{\alpha}^{\prime} s$ are rapidly decreasing functions. For a small $\varepsilon>0$, let $\psi_{\beta}(t) \in C^{\infty}\left(\mathbf{R}^{1}\right)$ be such that $\psi_{\beta}(t)=1$ if $t>\lambda-\varepsilon, \psi_{\beta}(t)=0$ if $t<\lambda-2 \varepsilon$. Let $\psi_{\beta}\left(D_{x_{\beta}}\right)$ be the pseudo differential operator with symbol $\psi_{\beta}\left(\left|\xi_{\beta}\right|^{2}\right)$. Fix $a>0$ arbitrarily. Then as $r=\left|x_{\beta}\right| \rightarrow \infty$, we have the following asymptotic expansion:

$$
\begin{aligned}
\psi_{\beta}\left(D_{x_{\beta}}\right) v & \simeq \sum_{l} C_{\beta l}(\lambda) u_{\beta l}\left(x^{\beta}\right) r^{-1} e^{i \sqrt{\lambda-E_{l}^{\beta}} r} A_{\beta l}\left(\lambda ; \theta_{\beta}, \omega\right), \theta_{\beta}=x_{\beta} / r, \\
C_{\beta l}(\lambda) & =2 \pi i\left(\lambda-E^{\alpha}\right)^{-1 / 4}\left(\lambda-E_{l}^{\beta}\right)^{-1 / 4},
\end{aligned}
$$

uniformly for $\left|x^{\beta}\right|<a, \theta_{\beta} \in S^{2}$.

One of the basic tools to prove the above theorems is the spectral representation theory developed for 2-body Schrödinger operators, the key idea of which is to relate the generalized eigenfunction to the spatial asymptotics of the resolvent of Schrödinger operators (see e.g. $[3,10]$ ). Another important tool is the estimate of the N-body resolvent ([2], Theorem 2.2) proved essentially by Skibsted [12], whose method is based on the study of propagation properties of the unitary group due to Sigal-Soffer [11]. This estimate has been further refined by Gérard [1].

Asymptotic properties of generalized eigenfunctions of three-body Schrödinger operators have been so far studied mainly by physicists. In the work of Newton [6], Theorems 1.1 and 1.3 were derived by intuitive arguments. If we consider the collision process of initial state of 3-clusters, we are led to consider the generalized eigenfunction formally defined by

$$
\psi(x, \lambda, \theta)=e^{i \sqrt{\lambda} \theta \cdot x}-R(\lambda+i 0) \sum_{\alpha} V_{\alpha} e^{i \sqrt{\lambda} \theta \cdot x} .
$$

The rigorous study of this generalized eigenfunction seems to be much harder. Nuttal [8] and Newton [6] gave precise explanations. For the structure of the related S-matrix, see e.g. [7]. One should also note the work of Mercuriev [5] of the three-body scattering theory for the Coulomb potential based on the stationary theory.

We shall quote freely our previous work [2], so the same notation is used in this paper. In particular, $L^{2, s}$ denotes the usual weighted $L^{2}$-space:

$$
f \in L^{2, s} \Leftrightarrow\|f\|_{s}^{2}=\int_{\mathbf{R}^{n}}\langle x\rangle^{2 s}|f(x)|^{2} d x<\infty,
$$

$\langle x\rangle=\left(1+|x|^{2}\right)^{1 / 2}$. For two Banach spaces $X_{1}$ and $X_{2}, \mathbf{B}\left(X_{1} ; X_{2}\right)$ denotes the totality of bounded operators from $X_{1}$ and $X_{2}$. 


\section{Proof of Theorem 1.1}

We recall the localization given in [2], 4-1. For a sufficiently small $\varepsilon>0$, we put

$$
\begin{aligned}
X_{\beta}^{\varepsilon} & =\left\{x \in X ;\left|x^{\beta}\right| /|x|<\varepsilon\right\}, \\
M^{\varepsilon} & =S^{5} \backslash \bigcup_{\beta} X_{\beta}^{\varepsilon}, \\
N^{\varepsilon} & =S^{5} \cap\left(\bigcup_{\beta} X_{\beta}^{\varepsilon}\right) .
\end{aligned}
$$

We take $\psi_{M}(\theta), \psi_{N}(\theta) \in C^{\infty}\left(S^{5}\right)$ such that

$$
\begin{gathered}
\psi_{M}(\theta)+\psi_{N}(\theta)=1, \\
\psi_{M}(\theta)= \begin{cases}1 & \theta \in M^{2 \varepsilon}, \\
0 & \theta \in N^{\varepsilon},\end{cases} \\
\psi_{N}(\theta)= \begin{cases}1 & \theta \in N^{\varepsilon}, \\
0 & \theta \in M^{2 \varepsilon}\end{cases}
\end{gathered}
$$

We also take $\chi_{M}(x), \chi_{N}(x) \in C^{\infty}(X)$ homogeneous of degree 0 for $|x|>1$ and

$$
\begin{aligned}
& \chi_{M}(x)= \begin{cases}1 & \text { if } \hat{x} \in M^{\varepsilon / 2},|x|>1, \\
0 & \text { if } \hat{x} \in N^{\varepsilon / 4},\end{cases} \\
& \chi_{N}(x)= \begin{cases}1 & \text { if } \hat{x} \in N^{2 \varepsilon},|x|>1, \\
0 & \text { if } \hat{x} \in M^{3 \varepsilon},\end{cases}
\end{aligned}
$$

where $\hat{x}=x /|x|$. Next we fix $\lambda>0$ arbitrarily and for a small $\varepsilon_{1}>0$ take $\psi_{1}(t) \in C_{0}^{\infty}\left(\mathbf{R}^{1}\right)$ such that

$$
\psi_{1}(t)= \begin{cases}1 & \text { if }|t-\lambda|<\varepsilon_{1} \\ 0 & \text { if }|t-\lambda|>2 \varepsilon_{1}\end{cases}
$$

Let $P$ be a Ps.D.Op. with symbol $p(x, \xi)=\chi_{M_{\hat{S}}}(x) \psi_{M}(\xi /|\xi|) \psi_{1}\left(|\xi|^{2}\right)$. Then, as has been proved in [2], (4.8), the kernel of $\psi_{M}(\theta) \hat{S}_{0 \alpha}(\lambda)$ is given by

$$
\begin{gathered}
-2 \pi i C_{\alpha}(\lambda) \mathscr{F}_{0}(\lambda)\left(H_{0}-\lambda\right) P^{*} R(\lambda+i 0) f, \\
f=\sum_{\gamma \neq \alpha} V_{\gamma}\left(x^{\gamma}\right) u_{\alpha}\left(x^{\alpha}\right) e^{i \sqrt{\lambda-E^{\alpha}} \omega \cdot x_{\alpha}} .
\end{gathered}
$$

We take $\chi(x) \in C^{\infty}(X)$ homogeneous of degree 0 for $|x|>1$ and

$$
\chi(x)= \begin{cases}1 & \text { if } \hat{x} \in M^{4 \varepsilon},|x|>1, \\ 0 & \text { if } \hat{x} \notin M^{3 \varepsilon} .\end{cases}
$$

To prove Theorem 1.1, we consider the limit of $\chi(x) v$ as $r=|x| \rightarrow \infty$. We introduce the following notation:

$$
u_{1} \sim u_{2} \Leftrightarrow r^{5 / 2}\left(u_{1}(r \cdot)-u_{2}(r \cdot)\right) \rightarrow 0 \quad \text { in } L^{2}\left(S^{5}\right) \text { as } r \rightarrow \infty .
$$


As can be checked easily, $w \sim 0$ if $w \in H^{1}\left(\mathbf{R}^{6}\right)$, where $H^{1}\left(\mathbf{R}^{6}\right)$ is the usual Sobolev space of order 1 .

Recall that $v=R(\lambda+i 0) f$ and that

$$
|f| \leqq C_{k}\left\langle x^{\alpha}\right\rangle^{-k}\left\langle x_{\alpha}\right\rangle^{-\rho}, \quad \forall k \geqq 0
$$

Let $\psi_{1}(t)$ be as above. Then $v-\psi_{1}(H) v \in H^{1}\left(\mathbf{R}^{6}\right)$. By [2], Lemma 2.1, $\chi(x)\left(\psi_{1}(H)-\psi_{1}\left(H_{0}\right)\right) v \in H^{1}\left(\mathbf{R}^{6}\right)$. So we have

$$
\chi(x) v \sim \chi(x) \psi_{1}\left(H_{0}\right) v .
$$

We set $\psi(\xi)=\psi_{1}\left(|\xi|^{2}\right) \psi_{M}(\xi /|\xi|)$. Since $\operatorname{supp} \chi$ and $\operatorname{supp}\left(1-\psi_{M}(\xi /|\xi|)\right) \psi_{1}\left(|\xi|^{2}\right)$ are disjoint, one can use [2], Theorem 2.2 to see

$$
\chi(x) \psi_{1}\left(H_{0}\right) v \sim \chi(x) \psi\left(D_{x}\right) v .
$$

Since $\chi(x)=\chi(x) \chi_{M}(x)$, we have

$$
\chi(x) \psi\left(D_{x}\right) v \sim \chi(x) \psi\left(D_{x}\right) \chi_{M}(x) v .
$$

In fact, on the support of the symbol of the commutator $\left[\chi_{M}(x), \psi\left(D_{x}\right)\right]$, the directions of $x$ and $\xi$ are different. So, (2.5) follows from [2], Theorem 2.2.

Noting that $P$ is a Ps.D.Op. with symbol $\chi_{M}(x) \psi(\xi)$, we let $g=\left(H_{0}-\lambda\right) P^{*} v$. We claim that $g \in L^{2, s}$ for some $s>3 / 2$. In fact, $g$ is written as

$$
g=\left[H_{0}, P^{*}\right] R(\lambda+i 0) f+P^{*} f-P^{*} V R(\lambda+i 0) f .
$$

As above, we apply [2], Theorem 2.2 to estimate the first term. By (2.2), the second and the third terms belong to $L^{2, s}$ for some $s>3 / 2$.

Lemma 2.1. Let $R_{0}(z)$ be the resolvent of $-\Delta$ in $\mathbf{R}^{n}$ and $f \in L^{2,3 / 2}$. Let $C(\lambda)=e^{(n-3) \pi i / 4} \pi^{-1 / 2} \lambda^{1 / 4}$. Then the following strong limit exists in $L^{2}\left(S^{n-1}\right)$ for any $\lambda>0$ :

$$
\mathscr{F}_{0}(\lambda) f=\mathrm{s}-\lim _{r \rightarrow \infty} C(\lambda) r^{(n-1) / 2} e^{-i \sqrt{\lambda} r}\left(R_{0}(\lambda+i 0) f\right)(r \cdot)
$$

where $\mathscr{F}_{0}(\lambda)$ is the Fourier transformation defined in the same way as in (1.6) with $C_{0}(\lambda)=(2 \pi)^{-n / 2} 2^{-1 / 2} \lambda^{(n-2) / 4}$. The right-hand side converges uniformly on $S^{n-1}$, if $f$ is rapidly decreasing.

Proof. For the proof, see e.g. [10]. The last assertion following from the asymptotic expansion of the Green's function of $-\Delta$.

We rewrite $P^{*} v$ as $P^{*} v=\left(H_{0}-\lambda-i 0\right)^{-1} g$ and apply the above lemma. Then

$$
\begin{aligned}
& P^{*} v \sim C(\lambda) r^{-5 / 2} e^{i \sqrt{\lambda} r} \mathscr{F}_{0}(\lambda)\left(H_{0}-\lambda\right) P^{*} v, \\
& C(\lambda)=e^{-3 \pi i / 4} \pi^{1 / 2} \lambda^{-1 / 4} .
\end{aligned}
$$

Equation (2.1) shows that

$$
\mathscr{F}_{0}(\lambda)\left(H_{0}-\lambda\right) P^{*} v=i(2 \pi)^{1 / 2} 2^{1 / 2}\left(\lambda-E^{\alpha}\right)^{-1 / 4} \psi_{M}(\theta) \hat{S}_{0 \alpha}(\lambda ; \theta, \omega) .
$$


In view of $(2.3) \sim(2.5)$ and (2.7), we have

$$
\begin{aligned}
& \chi(x) v \sim \chi(x) C_{1}(\lambda) r^{-5 / 2} e^{i \sqrt{\lambda} r} \psi_{M}(\theta) \hat{S}_{0 \alpha}(\lambda ; \theta, \omega), \\
& C_{1}(\lambda)=e^{-\pi i / 4} 2 \pi \lambda^{-1 / 4}\left(\lambda-E^{\alpha}\right)^{-1 / 4}, \quad \theta=x /|x| .
\end{aligned}
$$

This completes the proof of Theorem 1.1.

\section{Proof of Theorem 1.2}

We first recall the following well known fact.

Lemma 3.1. Let $T_{0}(\lambda)$ be defined by

$$
\left(T_{0}(\lambda) f\right)(\theta)=\int_{\mathbf{R}^{6}} e^{-i \sqrt{\lambda} \theta \cdot x} f(x) d x, \quad \theta \in S^{5} .
$$

Then for any $\lambda>0$, we have

$$
\left\|T_{0}(\lambda) f\right\|_{L^{2}\left(S^{5}\right)} \leqq C_{s}\|f\|_{s}, \quad s>1 / 2 .
$$

We next recall the localization given in [2], 4-3-1. Let $\psi_{N}, \psi_{1}$ be as in Sect. 2 and take $\psi_{\beta}(t) \in C_{0}^{\infty}\left(\mathbf{R}^{1}\right)$ such that

$$
\psi_{\beta}(t)= \begin{cases}1 & \text { if }|t-\lambda|<\varepsilon_{2}, \\ 0 & \text { if }|t-\lambda|>2 \varepsilon_{2} .\end{cases}
$$

Then by a suitable choice of $\varepsilon$ 's, we have

$$
\psi_{1}\left(|\xi|^{2}\right) \psi_{N}(\xi /|\xi|)=\sum_{\beta} \psi_{\beta}\left(\left|\xi_{\beta}\right|^{2}\right) \psi_{1}\left(|\xi|^{2}\right) \psi_{N}(\xi /|\xi|)
$$

We note that, if $\varepsilon$ is small, $\chi_{N}(x)$ is split into three parts:

$$
\begin{aligned}
& \chi_{N}(x)=\sum_{\beta} \chi_{\beta}(x), \\
& \chi_{\beta}(x)= \begin{cases}1 & \text { if } \hat{x} \in X_{\beta}^{2 \varepsilon}, \quad|x|>1, \\
0 & \text { if } \hat{x} \notin X_{\beta}^{3 \varepsilon} .\end{cases}
\end{aligned}
$$

Let $A_{\beta}$ be the Ps.D.Op. with symbol

$$
\chi_{\beta}(x) \psi_{\beta}\left(\left|\xi_{\beta}\right|^{2}\right) \psi_{1}\left(|\xi|^{2}\right) \psi_{N}(\xi /|\xi|) \text {. }
$$

Let $P=\sum_{\beta} A_{\beta}$. Then by [2], (4.19), in a small neighborhood of $N, \hat{S}_{0 \alpha}(\lambda ; \theta, \omega)$ is given by

$$
-2 \pi i C_{\alpha}(\lambda) \mathscr{F}_{0}\left[\left(H_{0}-\lambda\right) P^{*} R(\lambda+i 0) f\right](\lambda),
$$

where $f$ is defined by (1.12). It is easy to see that if $\theta$ is very close to $N \cap X_{\beta}$, $\hat{S}_{0 \alpha}(\lambda ; \theta, \omega)$ is represented as

$$
-2 \pi i C_{\alpha}(\lambda) \mathscr{F}_{0}\left[\left(H_{0}-\lambda\right) A_{\beta}^{*} R(\lambda+i 0) f\right](\lambda) .
$$

We take $\rho(t) \in C_{0}^{\infty}((0, \infty))$ such that $\int_{0}^{\infty} \rho(t) d t=1$. Let $v$ be defined by (1.11). 
Lemma 3.2. Let $\tilde{N}_{\beta}$ be a small neighborhood of $N \cap X_{\beta}$. Then the following strong limit exists in $L^{2}\left(\tilde{N}_{\beta}\right)$ :

$$
\mathrm{s}-\lim _{R \rightarrow \infty} \frac{1}{R} \int_{\mathbf{R}^{6}} e^{-i \sqrt{\lambda} \theta \cdot x} \theta_{\beta} \cdot \hat{x}_{\beta} \rho\left(\frac{\left|x_{\beta}\right|}{R}\right) A_{\beta}^{*} v d x,
$$

where $\hat{x}_{\beta}=x_{\beta} /\left|x_{\beta}\right|$.

The proof of this lemma is long and complicated. Let $\rho_{1}(t)=\int_{t}^{\infty} \rho(s) d s$ and $w=A_{\beta}^{*} v$. Then by integration by parts, we have

$$
\begin{aligned}
\frac{2 i \sqrt{\lambda}}{R} \int_{\mathbf{R}^{6}} e^{-i \sqrt{\lambda} \theta \cdot x} \theta_{\beta} \cdot \hat{x}_{\beta} \rho\left(\frac{\left|x_{\beta}\right|}{R}\right) w(x) d x= & \int_{\mathbf{R}^{6}} e^{-i \sqrt{\lambda} \theta \cdot x} \rho_{1}\left(\frac{\left|x_{\beta}\right|}{R}\right)(\Delta+\lambda) w(x) d x \\
& -\int_{\mathbf{R}^{6}} e^{-i \sqrt{\lambda} \theta \cdot x}\left(\Delta \rho_{1}\left(\frac{\left|x_{\beta}\right|}{R}\right)\right) \omega(x) d x .
\end{aligned}
$$

We show that the right-hand side of (3.4) converges in $L^{2}\left(\tilde{N}_{\beta}\right)$ as $R \rightarrow \infty$. It is easy to see that $\left(\Delta \rho_{1}\left(\left|x_{\beta}\right| / R\right)\right) w(x) \rightarrow 0$ in $L^{2, s}$ for some $s>1 / 2$ as $R \rightarrow \infty$. By Lemma 3.1 , the second term of the right-hand side of (3.4) tends to 0 in $L^{2}\left(S^{5}\right)$ as $R \rightarrow \infty$. The first term tends, formally, to

$$
\int_{\mathbf{R}^{6}} e^{-i \sqrt{\lambda} \theta \cdot x}(\Delta+\lambda) w(x) d x
$$

which coincides with $\hat{S_{0 \alpha}}(\lambda ; \theta, \omega)$ up to a constant depending on $\lambda$ by virtue of (3.2). But this term must be treated carefully, since $(\Delta+\lambda) w(x) \notin L^{2, s}$ for $s>1 / 2$.

Let $\Psi_{N}(\xi)=\psi_{1}\left(|\xi|^{2}\right) \psi_{N}(\xi /|\xi|)$ and $\psi_{\beta}\left(D_{x_{\beta}}\right)$ be the Ps.D.Op. with symbol $\psi_{\beta}\left(\left|\xi_{\beta}\right|^{2}\right)$. We set

$$
g_{\beta}=\left(H_{\beta}-\lambda\right) \psi_{\beta}\left(D_{x_{\beta}}\right) \chi_{\beta}(x) R(\lambda+i 0) f .
$$

By [2], Lemma $4.2, g_{\beta}$ is rapidly decreasing. A straightforward calculation shows that

$$
\begin{aligned}
(\Delta+\lambda) w & =-\sum_{\beta} \Psi_{N}\left(D_{x}\right)\left(H_{0}-\lambda\right) \psi_{\beta}\left(D_{x_{\beta}}\right) \chi_{\beta}(x) R(\lambda+i 0) f \\
& =-\sum_{\beta} \Psi_{N}\left(D_{x}\right) g_{\beta}+\sum_{\beta} \Psi_{N}\left(D_{x}\right) V_{\beta} R_{\beta}(\lambda+i 0) g_{\beta} .
\end{aligned}
$$

So, we have only to show the existence of the limit

$$
\mathrm{s}-\lim _{R \rightarrow \infty} \int_{\mathbf{R}^{6}} e^{-i \sqrt{\lambda} \theta \cdot x} \rho_{1}\left(\frac{\left|x_{\beta}\right|}{R}\right) V_{\beta} R_{\beta}(\lambda+i 0) g_{\beta} d x,
$$

in $L^{2}\left(\tilde{N}_{\beta}\right)$. Note that $\Psi_{N}(\sqrt{\lambda} \theta)=1$ if $\left|\theta^{\beta}\right|$ is sufficiently small. Let $r_{\beta}(z)=\left(h^{\beta}-z\right)^{-1}$. By the partial Fourier transformation with respect to $x_{\beta}$, $R_{\beta}(\lambda+i 0) g_{\beta}$ is transformed into $r_{\beta}\left(\lambda-\left|\xi_{\beta}\right|^{2}+i 0\right) \hat{g}_{\beta}$, where

$$
\hat{g}_{\beta}\left(x^{\beta}, \xi_{\beta}\right)=(2 \pi)^{-3 / 2} \int_{\mathbf{R}^{3}} e^{-i x_{\beta} \cdot \xi_{\beta}} g_{\beta}\left(x^{\beta}, x_{\beta}\right) d x_{\beta} .
$$


By the result of Jensen-Kato [4], the following expansion holds around $z=0$ :

$$
r_{\beta}(z)=\frac{B_{-2}}{z}+\frac{B_{-1}}{\sqrt{z}}+B_{0}(z),
$$

in $\mathbf{B}\left(L^{2, s} ; L^{2,-s}\right), s>5 / 2$, where $-B_{-2}=$ the projection onto the eigenspace of $h^{\beta}$ with zero eigenvalue, and $B_{-1}=-i B_{-2} V_{\beta} G V_{\beta} B_{-2}+i\left\langle\cdot, \varphi_{\beta}\right\rangle \varphi_{\beta}$, $G$ being an integral operator with kernel $\left|x^{\beta}-y^{\beta}\right|^{2} /(24 \pi)$, and $\varphi_{\beta}$ being the zero-resonance for $h^{\beta} . B_{0}(z)$ is a $\mathbf{B}\left(L^{2, s} ; L^{2,-s}\right)$-valued continuous function of $z$. We have, therefore,

$$
\begin{aligned}
R_{\beta}(\lambda+i 0) g_{\beta}= & (2 \pi)^{-3 / 2} \int_{\mathbf{R}^{3}} e^{i x_{\beta} \cdot \xi_{\beta}} \frac{B_{-2} \hat{g}_{\beta}}{\lambda-\left|\xi_{\beta}\right|^{2}+i 0} d \xi_{\beta} \\
& +(2 \pi)^{-3 / 2} \int_{\mathbf{R}^{3}} e^{i x_{\beta} \cdot \xi_{\beta}} \frac{B_{-1} \hat{g}_{\beta}}{\sqrt{\lambda-\left|\xi_{\beta}\right|^{2}+i 0}} d \xi_{\beta} \\
& +(2 \pi)^{-3 / 2} \int_{\mathbf{R}^{3}} e^{i x_{\beta} \cdot \xi_{\beta}} B_{0}\left(\lambda-\left|\xi_{\beta}\right|^{2}+i 0\right) \hat{g}_{\beta} d \xi_{\beta} .
\end{aligned}
$$

Now we consider the limit (3.6). Let $u_{\beta}^{(j)}$ be normalized eigenfunctions of $h^{\beta}$ with zero eigenvalue, and put

$$
\tilde{g}_{\beta j}\left(\xi_{\beta}\right)=\int_{\mathbf{R}^{3}} \overline{u_{\beta}^{(j)}\left(x^{\beta}\right)} \hat{g}_{\beta}\left(x^{\beta}, \xi_{\beta}\right) d x^{\beta}
$$

Then we have

$$
B_{-2} \hat{g}_{\beta}=-\sum_{j} u_{\beta}^{(j)}\left(x^{\beta}\right) \tilde{g}_{\beta j}\left(\xi_{\beta}\right)
$$

So, letting $r_{0}(z)=\left(-\Delta_{x_{\beta}}-z\right)^{-1}$ and $g_{\beta j}$ be the inverse Fourier transform of $\tilde{g}_{\beta j}$, we have

$$
(2 \pi)^{-3 / 2} \int_{\mathbf{R}^{3}} e^{i x_{\beta} \cdot \xi_{\beta}} \frac{B_{-2} \hat{g}_{\beta}}{\lambda-\left|\xi_{\beta}\right|^{2}+i 0} d \xi_{\beta}=\sum_{j} u_{\beta}^{(j)}\left(x^{\beta}\right) r_{0}(\lambda+i 0) g_{\beta j} .
$$

Lemma 3.3. The following strong limit exists in $L^{2}\left(\tilde{N}_{\beta}\right)$ :

$$
\mathrm{s}-\lim _{R \rightarrow \infty} \int_{\mathbf{R}^{3}} e^{-i \sqrt{\lambda} \theta \cdot x} \rho_{1}\left(\frac{\left|x_{\beta}\right|}{R}\right) V_{\beta}\left(x^{\beta}\right) u_{\beta}^{(j)}\left(x^{\beta}\right)\left(r_{0}(\lambda+i 0) g_{\beta j}\right)\left(x_{\beta}\right) d x .
$$

Proof. We first rewrite the above integral as

$$
\int_{\mathbf{R}^{3}} e^{-i \sqrt{\lambda} \theta^{\beta} \cdot x^{\beta}} V_{\beta}\left(x^{\beta}\right) u_{\beta}^{(j)}\left(x^{\beta}\right) d x^{\beta} \times \int_{\mathbf{R}^{3}} e^{-i \sqrt{\lambda} \theta_{\beta} \cdot x_{\beta}} \rho_{1}\left(\frac{\left|x_{\beta}\right|}{R}\right)\left(r_{0}(\lambda+i 0) g_{\beta j}\right)\left(x_{\beta}\right) d x_{\beta} .
$$

In the following arguments, we always assume that $\left|\theta^{\beta}\right| \neq 0$ and $\left|\theta^{\beta}\right|$ is sufficiently small. Taking account of the relation

$$
(-\Delta-\lambda) e^{-i \sqrt{\lambda} \theta_{\beta} \cdot x_{\beta}}=-\lambda\left|\theta^{\beta}\right|^{2} e^{-i \sqrt{\lambda} \theta_{\beta} \cdot x_{\beta}},
$$


and letting

$$
a\left(\theta^{\beta}\right)=\frac{1}{-\lambda\left|\theta^{\beta}\right|^{2}} \int_{\mathbf{R}^{3}} e^{-i \sqrt{\lambda} \theta^{\beta} \cdot x^{\beta}} V_{\beta}\left(x^{\beta}\right) u_{\beta}^{(j)}\left(x^{\beta}\right) d x^{\beta},
$$

one can rewrite $(3.10)$ as

$$
\begin{aligned}
& a\left(\theta^{\beta}\right) \int_{\mathbf{R}^{3}}\left((-\Delta-\lambda) e^{-i \sqrt{\lambda} \theta_{\beta} \cdot x_{\beta}}\right) \rho_{1}\left(\frac{\left|x_{\beta}\right|}{R}\right) r_{0}(\lambda+i 0) g_{\beta j} d x_{\beta} \\
& =a\left(\theta^{\beta}\right)\left\{-\int_{\mathbf{R}^{3}} e^{-i \sqrt{\lambda} \theta_{\beta} \cdot x_{\beta}}\left(\Delta \rho_{1}\left(\frac{\left|x_{\beta}\right|}{R}\right)\right) r_{0}(\lambda+i 0) g_{\beta j} d x_{\beta}\right. \\
& +\frac{2}{R} \int_{\mathbf{R}^{3}} e^{-i \sqrt{\lambda} \theta_{\beta} \cdot x_{\beta}} \rho\left(\frac{\left|x_{\beta}\right|}{R}\right) \frac{\partial}{\partial\left|x_{\beta}\right|} r_{0}(\lambda+i 0) g_{\beta j} d x_{\beta} \\
& \left.+\int_{\mathbf{R}^{3}} e^{-i \sqrt{\lambda} \theta_{\beta} \cdot x_{\beta}} \rho_{1}\left(\frac{\left|x_{\beta}\right|}{R}\right) g_{\beta j} d x_{\beta}\right\} .
\end{aligned}
$$

Let $K\left(\theta^{\beta}\right)$ be the sphere:

$$
K\left(\theta^{\beta}\right)=\left\{\theta_{\beta} ;\left|\theta_{\beta}\right|=\sqrt{1-\left|\theta^{\beta}\right|^{2}}\right\} .
$$

By [2], Lemma 2.3, $\left|a\left(\theta^{\beta}\right)\right| \leqq C\left|\theta^{\beta}\right|^{-1}$. Hence, to show the convergence of (3.11) in $L^{2}\left(\tilde{N}_{\beta}\right)$, we have only to show that each term in the parenthesis $\{\ldots\}$ converges in $L^{2}\left(K\left(\theta^{\beta}\right)\right)$ and the convergence is uniform for small $\left|\theta^{\beta}\right|$. This is obvious for the third term, since $g_{\beta j}$ is rapidly decreasing. It is easy to see that $\left(\Delta \rho_{1}\left(\left|x_{\beta}\right| / R\right)\right) r_{0}(\lambda+i 0) g_{\beta j} \rightarrow 0$ in $L^{2, s}$ for some $s>1 / 2$. By Lemma 3.1, the first term converges in $L^{2}\left(K\left(\theta^{\beta}\right)\right)$ uniformly for small $\left|\theta^{\beta}\right|$. A simple computation shows that $\left(\frac{\partial}{\partial\left|x_{\beta}\right|}-i \sqrt{\lambda}\right) r_{0}(\lambda+i 0) g_{\beta j}=O\left(\left|x_{\beta}\right|^{-2}\right)$, hence

$$
R^{-1} \rho\left(\left|x_{\beta}\right| / R\right)\left(\frac{\partial}{\partial\left|x_{\beta}\right|}-i \sqrt{\lambda}\right) r_{0}(\lambda+i 0) g_{\beta j} \rightarrow 0
$$

in $L^{2, s}$ for some $s>1 / 2$. So, it remains to consider

$$
\frac{1}{R} \int_{\mathbf{R}^{3}} e^{-i \sqrt{\lambda} \theta_{\beta} \cdot x_{\beta}} \rho\left(\frac{\left|x_{\beta}\right|}{R}\right) r_{0}(\lambda+i 0) g_{\beta j} d x_{\beta} .
$$

By the well-known property of the Green's function of $-\Delta_{x_{\beta}}$, we have

$$
r_{0}(\lambda+i 0) g_{\beta j}=C(\lambda) \frac{e^{i \sqrt{\lambda}\left|x_{\beta}\right|}}{\left|x_{\beta}\right|} \psi(\omega)+O\left(\left|x_{\beta}\right|^{-2}\right),
$$

where $\psi(\omega)=\hat{g}_{\beta j}(\sqrt{\lambda} \omega), \omega=x_{\beta} /\left|x_{\beta}\right|$. Then, up to a constant, (3.12) is asymptotically equal to

$$
\begin{aligned}
& \frac{1}{R} \int_{\mathbf{R}^{3}} e^{-i \sqrt{\lambda} \theta_{\beta} \cdot x_{\beta}} \rho\left(\frac{\left|x_{\beta}\right|}{R}\right) \frac{e^{i \sqrt{\lambda}\left|x_{\beta}\right|}}{\left|x_{\beta}\right|} \psi(\omega) d x_{\beta} \\
& =R \int_{0}^{\infty} e^{i \sqrt{\lambda} R t} \rho(t) t d t \int_{S^{2}} e^{-i \sqrt{\lambda} R t \theta_{\beta} \cdot \omega} \psi(\omega) d \omega .
\end{aligned}
$$


The stationary phase method on the sphere and integration by parts show that (3.13) is asymptotically equal to, up to a constant,

$$
\frac{1}{\left|\theta_{\beta}\right|} \int_{0}^{\infty} e^{i \sqrt{\lambda} R t\left(1-\left|\theta_{\beta}\right|\right)} \rho(t) d t \times \psi\left(\theta_{\beta} /\left|\theta_{\beta}\right|\right) .
$$

Now, it is easy to see that (3.14) is convergent as $R \rightarrow \infty$.

We next consider

$$
\int_{\mathbf{R}^{6}} e^{-i \sqrt{\lambda} \theta \cdot x} \rho_{1}\left(\frac{\left|x_{\beta}\right|}{R}\right) V_{\beta}\left(x^{\beta}\right)\left(\int_{\mathbf{R}^{3}} e^{i x_{\beta} \cdot \xi_{\beta}} \frac{B_{-1} \hat{g}_{\beta}}{\sqrt{\lambda-\left|\xi_{\beta}\right|^{2}+i 0}} d \xi_{\beta}\right) d x,
$$

which corresponds to the second term of the right-hand side of (3.8). Note that by virtue of [2], Lemma $2.3, B_{-1} \hat{g}_{\beta}$ consists of a sum of the terms, $\psi\left(x^{\beta}\right) f\left(\xi_{\beta}\right)$, where $\left|\psi\left(x^{\beta}\right)\right| \leqq C\left\langle x^{\beta}\right\rangle^{-1}$, and $f\left(\xi_{\beta}\right)$ is a smooth function supported near the shell $\left|\xi_{\beta}\right|=\sqrt{\lambda}$.

Lemma 3.4. Let $\left|\psi\left(x^{\beta}\right)\right| \leqq C\left\langle x^{\beta}\right\rangle^{-1}$, and $f\left(\xi_{\beta}\right)$ be a smooth function supported near the shell $\left|\xi_{\beta}\right|=\sqrt{\lambda}$. Then there exists the following strong limit in $L^{2}\left(\tilde{N}_{\beta}\right)$ :

$$
\mathrm{s}-\lim _{\boldsymbol{R} \rightarrow \infty} \int_{\mathbf{R}^{6}} e^{-i \sqrt{\lambda} \theta \cdot x} \rho_{1}\left(\frac{\left|x_{\beta}\right|}{R}\right) V_{\beta}\left(x^{\beta}\right) \psi\left(x^{\beta}\right)\left(\int_{\mathbf{R}^{3}} e^{i x_{\beta} \cdot \xi_{\beta}} \frac{f\left(\xi_{\beta}\right)}{\sqrt{\lambda-\left|\xi_{\beta}\right|^{2}+i 0}} d \xi_{\beta}\right) d x .
$$

Proof. We have only to consider the integral

$$
\int_{\mathbf{R}^{3}} e^{-i \sqrt{\lambda} \theta_{\beta} \cdot x_{\beta}} \rho_{1}\left(\frac{\left|x_{\beta}\right|}{R}\right)\left(\int_{\mathbf{R}^{3}} e^{i x_{\beta} \cdot \xi_{\beta}} \frac{f\left(\xi_{\beta}\right)}{\sqrt{\lambda-\left|\xi_{\beta}\right|^{2}+i 0}} d \xi_{\beta}\right) d x_{\beta} .
$$

We calculate the limit as $R \rightarrow \infty$ by the repeated application of the method of stationary phase. We first integrate in $\omega=\xi_{\beta} /\left|\xi_{\beta}\right|$. Then letting $r=\left|x_{\beta}\right|$ and $\omega_{x}=x_{\beta} / r$, we have as $r \rightarrow \infty$,

$$
\begin{aligned}
\int_{S^{2}} e^{i x_{\beta} \cdot\left|\xi_{\beta}\right| \omega} f\left(\left|\xi_{\beta}\right| \omega\right) d \omega \sim & e^{i r\left|\xi_{\beta}\right|} \sum_{n \geqq 0} r^{-1-n} f_{+n}\left(\left|\xi_{\beta}\right|, \omega_{x}\right) \\
& +e^{-i r\left|\xi_{\beta}\right|} \sum_{n \geqq 0} r^{-1-n} f_{-n}\left(\left|\xi_{\beta}\right|, \omega_{x}\right) .
\end{aligned}
$$

We concentrate on calculating the contribution of the first term $e^{i r\left|\xi_{\beta}\right|} r^{-1} f_{+0}\left(\left|\xi_{\beta}\right|\right.$, $\omega_{x}$ ). To perform the integration in $\left|\xi_{\beta}\right|$, we split the integral into two parts: $\left\{\left|\xi_{\beta}\right| \geqq \sqrt{\lambda}\right\}$ and $\left\{\left|\xi_{\beta}\right| \leqq \sqrt{\lambda}\right\}$, and compute on the region $\left\{\left|\xi_{\beta}\right| \geqq \sqrt{\lambda}\right\}$ for example. Then letting $\left|\xi_{\beta}\right|-\sqrt{\lambda}=t^{2}, t>0$, and

$$
f_{1}\left(t^{2}, \omega_{x}\right)=2 f_{+0}\left(t^{2}+\sqrt{\lambda}, \omega_{x}\right)\left(t^{2}+2 \sqrt{\lambda}\right)^{-1 / 2}\left(t^{2}+\sqrt{\lambda}\right)^{2},
$$

we have

$$
\int_{\sqrt{\lambda}}^{\infty} e^{i r\left|\xi_{\beta}\right|} r^{-1} f_{+0}\left(\left|\xi_{\beta}\right|, \omega_{x}\right) \frac{\left|\xi_{\beta}\right|^{2}}{\sqrt{\left|\xi_{\beta}\right|^{2}-\lambda}} d\left|\xi_{\beta}\right|=e^{i \sqrt{\lambda} r_{r}} r^{-1} \int_{0}^{\infty} e^{i r t^{2}} f_{1}\left(t^{2}, \omega_{x}\right) d t .
$$

By the stationary phase method, we have as $r \rightarrow \infty$,

$$
e^{i \sqrt{\lambda} r} r^{-1} \int_{0}^{\infty} e^{i r t^{2}} f_{1}\left(t^{2}, \omega_{x}\right) d t \sim e^{i \sqrt{\lambda} r} \sum_{n \geqq 0} r^{-3 / 2-n} g_{n}\left(\omega_{x}\right) .
$$


So, we consider the contribution of the first term $e^{i \sqrt{\lambda} r} r^{-3 / 2} g_{0}\left(\omega_{x}\right)$. By integrating in $\omega_{x}$, we have as $r \rightarrow \infty$,

$$
\begin{aligned}
\int_{S^{2}} e^{-i \sqrt{\lambda} r \omega_{x^{*}} \cdot \theta_{\theta}} e^{i \sqrt{\lambda} r} r^{-3 / 2} g_{0}\left(\omega_{x}\right) d \omega_{x} \sim e^{i \sqrt{\lambda} r\left(1-\left|\theta_{\theta}\right|\right)} \sum_{n \geqq 0} r^{-5 / 2-n} h_{+n}\left(\left|\theta_{\beta}\right|, \omega_{\theta}\right) \\
+e^{i \sqrt{\lambda} r\left(1+\left|\theta_{\theta}\right|\right)} \sum_{n \geqq 0} r^{-5 / 2-n} h_{-n}\left(\left|\theta_{\beta}\right|, \omega_{\theta}\right),
\end{aligned}
$$

where $\omega_{\theta}=\theta_{\beta} /\left|\theta_{\beta}\right|$. We look at the first term $e^{i \sqrt{\lambda} r\left(1-\left|\theta_{\theta}\right|\right)} r^{-5 / 2} h_{+0}\left(\left|\theta_{\beta}\right|, \omega_{\theta}\right)$. By integrating in $r$, we have

$$
\begin{aligned}
& \int_{0}^{\infty} e^{i \sqrt{\lambda} r\left(1-\left|\theta_{\theta}\right|\right)} r^{-5 / 2} h_{+0}\left(\left|\theta_{\beta}\right|, \omega_{\theta}\right) \rho_{1}\left(\frac{r}{R}\right) r^{2} d r \\
= & h_{+0}\left(\left|\theta_{\beta}\right|, \omega_{\theta}\right) \times 2 \sqrt{R} \int_{0}^{\infty} e^{i R \sqrt{\lambda}\left(1-\left|\theta_{\beta}\right|\right) t^{2}} \rho_{1}\left(t^{2}\right) d t .
\end{aligned}
$$

Here we note the inequality

$$
\left|\int_{-\infty}^{\infty} e^{i k t^{2}} f(t) d t\right| \leqq \frac{C}{\sqrt{k}},
$$

for $f \in C_{0}^{\infty}\left(\mathbf{R}^{1}\right)$ and any $k>0$, which follows from the stationary phase method. Therefore, we have

$$
\left|\sqrt{R} \int_{0}^{\infty} e^{i R \sqrt{\lambda}\left(1-\left|\theta_{\beta}\right|\right) t^{2}} \rho_{1}\left(t^{2}\right) d t\right| \leqq C\left(1-\left|\theta_{\beta}\right|\right)^{-1 / 2} \leqq C\left|\theta^{\beta}\right|^{-1} .
$$

This shows that (3.17) is dominated by $C\left|\theta^{\beta}\right|^{-1}$ uniformly in $\left|\theta_{\beta}\right|$ and $R$. One can also see by the stationary phase method that (3.17) is convergent pointwise as $R \rightarrow \infty$, when $\left|\theta_{\beta}\right|<1$. These two observations prove that (3.17) converges in $L^{2}\left(\tilde{N}_{\beta}\right)$ as $R \rightarrow \infty$. In a similar and simpler way, one can treat all the terms of the asymptotic expansion appearing above. Hence (3.16) converges in $L^{2}\left(\tilde{N}_{\beta}\right)$ as $R \rightarrow \infty$.

We finally consider the limit corresponding to the last term in (3.8).

Lemma 3.5. The following strong limit exists in $L^{2}\left(\tilde{N}_{\beta}\right)$ :

$$
\mathrm{s}-\lim _{R \rightarrow \infty} \int_{\mathbf{R}^{6}} e^{-i \sqrt{\lambda} \theta \cdot x} \rho_{1}\left(\frac{\left|x_{\beta}\right|}{R}\right) V_{\beta}\left(x^{\beta}\right)\left(\int_{\mathbf{R}^{3}} e^{i x_{\beta} \cdot \xi_{\beta}} B_{0}\left(\lambda-\left|\xi_{\beta}\right|^{2}+i 0\right) \hat{g}_{\beta} d \xi_{\beta}\right) d x .
$$

Proof. Let

$$
a\left(\xi_{\beta}\right)=\int_{\mathbf{R}^{3}} e^{-i \sqrt{\lambda} \theta^{\beta} \cdot x^{\beta}} V_{\beta}\left(x^{\beta}\right)\left(B_{0}\left(\lambda-\left|\xi_{\beta}\right|^{2}+i 0\right) \hat{g}_{\beta}\right)\left(x^{\beta}, \xi_{\beta}\right) d x^{\beta} .
$$

Then $a\left(\xi_{\beta}\right)$ is a continuous function of compact support. Letting $\tilde{\rho}_{1}\left(\xi_{\beta}\right)$ be the inverse Fourier transform of $\rho_{1}\left(\left|x_{\beta}\right|\right)$, one can rewrite the integral in question as follows:

$$
\int_{\mathbf{R}^{3}} \tilde{\rho}_{1}\left(\eta_{\beta}\right) a\left(\sqrt{\lambda} \theta_{\beta}+\frac{\eta_{\beta}}{R}\right) d \eta_{\beta},
$$

from which the lemma follows immediately. 
Lemma 3.2 now follows from Lemmas 3.3, 3.4 and 3.5.

Our next aim is to rewrite (3.3). Since $R^{-1}\left[\rho\left(\left|x_{\beta}\right| / R\right), A_{\beta}^{*}\right] v \rightarrow 0$ in $L^{2, s}$ for some $s>1 / 2$, it follows from Lemma 3.1 that as $R \rightarrow \infty$, the left-hand side of (3.3) is asymptotically equal to

$$
\frac{1}{R} \int_{\mathbf{R}^{6}} e^{-i \sqrt{\lambda} \theta \cdot x} \theta_{\beta} \cdot \hat{x}_{\beta} A_{\beta}^{*} \rho\left(\frac{\left|x_{\beta}\right|}{R}\right) v d x .
$$

We take a bounded $C^{\infty}$-function $a_{1}\left(x_{\beta}, \xi_{\beta}\right)$ such that $a_{1}\left(x_{\beta}, \xi_{\beta}\right)=\hat{x}_{\beta} \cdot \xi_{\beta} / \sqrt{\lambda}$ when $\left|x_{\beta}\right|>1$ and $\left|\xi_{\beta}\right|$ is close to $\sqrt{\lambda}$. Let $A_{1}$ be the Ps.D.Op. with symbol $a_{1}\left(x_{\beta}, \xi_{\beta}\right)$. Let $T_{0}(\lambda)$ be as in Lemma 3.1. Then (3.18) is asymptotically equal to $R^{-1} T_{0}(\lambda) A_{1}^{*} A_{\beta}^{*} \rho\left(\left|x_{\beta}\right| / R\right) v$.

Let $P_{1}$ be the Ps.D.Op. with symbol

$$
a_{1}\left(x_{\beta}, \xi_{\beta}\right) \chi_{\beta}(x) \psi_{\beta}\left(\left|\xi_{\beta}\right|^{2}\right) \psi_{1}\left(|\xi|^{2}\right) \psi_{N}(\xi /|\xi|) \text {. }
$$

Then, since

$$
\frac{1}{R}\left(A_{1}^{*} A_{\beta}^{*}-P_{1}^{*}\right) \rho\left(\frac{\left|x_{\beta}\right|}{R}\right) v \rightarrow 0
$$

in $L^{2, s}$ for some $s>1 / 2,(3.18)$ is asymptotically equal to

$$
\frac{1}{R} \int_{\mathbf{R}^{6}} e^{-i \sqrt{\lambda} \theta \cdot x} \theta_{\beta} \cdot \hat{x}_{\beta} \chi_{\beta}(x) \rho\left(\frac{\left|x_{\beta}\right|}{R}\right) v(x) d x .
$$

We have localized $v$ in the region $\left\{x ; a<\left|x_{\beta}\right| / R<b,\left|x^{\beta}\right| \leqq 3 \varepsilon|x|\right\}$. The next aim is to localize $v$ in the region where $\theta_{\beta}$ and $x_{\beta}$ have almost the same directions.

Theorem 3.6. Let $P_{\beta}$ be the Ps.D.Op. with symbol $p\left(x_{\beta}, \xi_{\beta}\right)$ having the following properties:

$$
\left|\partial_{x_{\beta}}^{m} \partial_{\xi_{\beta}}^{n} p\left(x_{\beta}, \xi_{\beta}\right)\right| \leqq C_{m n}\left\langle x_{\beta}\right\rangle^{-m} \quad \forall m, n,
$$

there exist constants $0<a<b<\infty$ such that

$$
\operatorname{supp}_{\xi_{\beta}} p\left(x_{\beta}, \xi_{\beta}\right) \subset\left\{a<\left|\xi_{\beta}\right|<b\right\},
$$

there exists a constant $\mu_{-}$such that $-1<\mu_{-}<1$ and

$$
p\left(x_{\beta}, \xi_{\beta}\right)=0 \text { if } x_{\beta} \cdot \xi_{\beta}>\mu_{-}\left|x_{\beta}\right|\left|\xi_{\beta}\right| .
$$

Let $\chi_{\beta}(x)$ be as above. Suppose that $V_{\alpha}$ satisfies (1.9) with $\rho>0$. Then we have

$$
\langle x\rangle^{s} P_{\beta} \chi_{\beta}(x) R(\lambda+i 0)\langle x\rangle^{-s-t} \in \mathbf{B}\left(L^{2}(X) ; L^{2}(X)\right),
$$

for $s>-1 / 2, t>1, \lambda \in \sigma_{e}(H) \backslash \Lambda$.

We prove this theorem in the next section.

Let $\rho_{+}(t) \in C^{\infty}\left(\mathbf{R}^{1}\right)$ be such that $\rho_{+}(t)=1$ for $t>1-\varepsilon_{3}, \rho_{+}(t)=0$ for $t<1-2 \varepsilon_{3}, \varepsilon_{3}$ being a sufficiently small constant. Let $\rho_{-}(t)=1-\rho_{+}(t)$. We take $b\left(x_{\beta}, \xi_{\beta}\right) \in C^{\infty}$ such that

$$
b\left(x_{\beta}, \xi_{\beta}\right)=\rho_{-}\left(\frac{\xi_{\beta}}{\left|\xi_{\beta}\right|} \cdot \frac{x_{\beta}}{\left|x_{\beta}\right|}\right)
$$


if $\left|x_{\beta}\right|>1$ and $\left|\xi_{\beta}\right|>\sqrt{\lambda} / 2$. Let $P_{\beta}$ be the Ps.D.Op. with symbol $b\left(x_{\beta}, \xi_{\beta}\right)$. Let $P_{1}$ be as above. Then by Theorem 3.6, we have

$$
\frac{1}{R} P_{1}^{*} P_{\beta}^{*} \rho\left(\frac{\left|x_{\beta}\right|}{R}\right) v \rightarrow 0,
$$

in $L^{2, s}$ for some $s>1 / 2$. Multiplying $T_{0}(\lambda)$ and looking at the symbol of $P_{1}^{*} P_{\beta}^{*}$, we have

$$
\frac{1}{R} \int_{\mathbf{R}^{6}} e^{-i \sqrt{\lambda} \theta \cdot x} \theta_{\beta} \cdot \hat{x}_{\beta} \rho_{-}\left(\frac{\theta_{\beta}}{\left|\theta_{\beta}\right|} \cdot \frac{x_{\beta}}{\left|x_{\beta}\right|}\right) \chi_{\beta}(x) \rho\left(\frac{\left|x_{\beta}\right|}{R}\right) v d x \rightarrow 0
$$

as $R \rightarrow \infty$. By (3.19) and (3.24), we have shown

Lemma 3.7. The following strong limit exists in $L^{2}\left(\tilde{N}_{\beta}\right)$ :

$$
\mathrm{s}-\lim _{R \rightarrow \infty} \frac{1}{R} \int_{\mathbf{R}^{6}} e^{-i \sqrt{\lambda} \theta \cdot x} \theta_{\beta} \cdot \hat{x}_{\beta} \rho_{+}\left(\frac{\theta_{\beta}}{\left|\theta_{\beta}\right|} \cdot \frac{x_{\beta}}{\left|x_{\beta}\right|}\right) \chi_{\beta}(x) \rho\left(\frac{\left|x_{\beta}\right|}{R}\right) v(x) d x .
$$

The proof of Theorem 1.2 is now completed if we show that (3.25) coincides with $C_{2}(\lambda) \hat{S}_{0 \alpha}(\lambda ; \theta, \omega)$. For this purpose, it is sufficient to show that for any $\psi(\theta) \in C_{0}^{\infty}\left(\tilde{N}_{\beta}-X_{\beta}\right)$,

$$
\begin{gathered}
\lim _{R \rightarrow \infty} \frac{1}{R} \int_{\mathbf{R}^{6}} \int_{S^{5}} \psi(\theta) e^{-i \sqrt{\lambda} \theta \cdot x} \theta_{\beta} \cdot \hat{x}_{\beta} \rho_{+}\left(\frac{\theta_{\beta}}{\left|\theta_{\beta}\right|} \cdot \frac{x_{\beta}}{\left|x_{\beta}\right|}\right) \chi_{\beta}(x) \rho\left(\frac{\left|x_{\beta}\right|}{R}\right) v(x) d x d \theta \\
=C_{2}(\lambda) \int_{S^{5}} \psi(\theta) \hat{S}_{0 \alpha}(\lambda ; \theta, \omega) d \theta .
\end{gathered}
$$

Applying the stationary phase method on the sphere and taking into account of the fact that on the support of the integrand $\theta_{\beta}, x_{\beta}$ are sufficiently close to $\theta$ and $x$, we have as $r=|x| \rightarrow \infty$,

$$
\begin{gathered}
\int_{S^{5}} \psi(\theta) e^{-i \sqrt{\lambda} \theta \cdot x} \theta_{\beta} \cdot \hat{x}_{\beta} \rho_{+}\left(\frac{\theta_{\beta}}{\left|\theta_{\beta}\right|} \cdot \frac{x_{\beta}}{\left|x_{\beta}\right|}\right) \chi_{\beta}(x) d \theta \sim C_{3}(\lambda) e^{-i \sqrt{\lambda} r}\left|x_{\beta}\right| r^{-7 / 2} \psi(x / r), \\
C_{3}(\lambda)=e^{5 \pi i / 4}(2 \pi)^{5 / 2} \lambda^{-5 / 4},
\end{gathered}
$$

where we have used the fact that $\chi_{\beta}(x)=1$ if $r>1$ and $\psi(x / r) \neq 0$.

On the support of $\psi(x / r), x / r \in M$. Therefore by Theorem 1.1 , we have

$$
e^{-i \sqrt{\lambda} r}\left|x_{\beta}\right| r^{-7 / 2} \psi(x / r) \rho\left(\left|x_{\beta}\right| / R\right) v \sim C_{1}(\lambda)\left|x_{\beta}\right| r^{-6} \psi(x / r) \rho\left(\left|x_{\beta}\right| / R\right) \hat{S}_{0 \alpha}(\lambda ; x / r, \omega),
$$

as $R \rightarrow \infty$. Hence we have

$$
\begin{aligned}
\lim _{R \rightarrow \infty} & \frac{1}{R} \int_{\mathbf{R}^{6}} \int_{S^{5}} \psi(\theta) e^{-i \sqrt{\lambda} \theta \cdot x} \theta_{\beta} \cdot \hat{x}_{\beta} \rho_{+}\left(\frac{\theta_{\beta}}{\left|\theta_{\beta}\right|} \cdot \frac{x_{\beta}}{\left|x_{\beta}\right|}\right) \chi_{\beta}(x) \rho\left(\frac{\left|x_{\beta}\right|}{R}\right) v(x) d x d \theta \\
& =\lim _{R \rightarrow \infty} C_{1}(\lambda) C_{3}(\lambda) \frac{1}{R} \int_{\mathbf{R}^{6}}\left|x_{\beta}\right| r^{-6} \psi(x / r) \rho\left(\left|x_{\beta}\right| / R\right) \hat{S}_{0 \alpha}(\lambda ; x / r, \omega) d x .
\end{aligned}
$$

Passing to the polar coordinates $x=r \theta$, we have

$$
\frac{1}{R} \int_{S^{5}} \int_{0}^{\infty}\left|\theta_{\beta}\right| \psi(\theta) \rho\left(\left|\theta_{\beta}\right| r / R\right) \hat{S}_{0 \alpha}(\lambda ; \theta, \omega) d \theta d r=\int_{S^{5}} \psi(\theta) \hat{S}_{0 \alpha}(\lambda ; \theta, \omega) d \theta
$$


where we have used the substitution $t=\left|\theta_{\beta}\right| r / R$ and $\int_{0}^{\infty} \rho(t) d t=1$. This proves (3.26).

\section{Micro-Local Positivities and Resolvent Estimates}

We explain the proof of Theorem 3.6. To make the arguments clear we first explain it in the case of the 2-body Schrödinger operators. We introduce the following class of symbols:

$$
\begin{aligned}
& p(x, \xi) \in S_{-}^{m} \Leftrightarrow\left\{\begin{array}{l}
\left|\partial_{x}^{\alpha} \partial_{\xi}^{\beta} p(x, \xi)\right| \leqq C_{\alpha \beta}\langle x\rangle^{m-|\alpha|}, \text { there exist constants } \\
0<a<b<\infty \text { such that } \operatorname{supp}_{\xi} p(x, \xi) \subset\{a<|\xi|<b\}, \\
p(x, \xi)=0 \text { if } \hat{x} \cdot \hat{\xi}>1-\varepsilon(0<\varepsilon<1) .
\end{array}\right. \\
& p(x, \xi) \in S^{-N} \Leftrightarrow\left|\partial_{x}^{\alpha} \partial_{\xi}^{\beta} p(x, \xi)\right| \leqq C_{\alpha \beta}\langle x\rangle^{-N-|\alpha|} .
\end{aligned}
$$

Here and in the sequel, $\hat{x}=x /|x|$ and $\hat{\xi}=\xi /|\xi|$.

$p(x, \xi) \in S_{-}^{2 m}$ is said to be a symbol of canonical type if

$$
p(x, \xi)=(|x||\xi|-x \cdot \xi)^{2 m} \rho(\hat{x} \cdot \hat{\xi}) \varphi(\xi) \chi(x),
$$

where $\rho(t) \geqq 0, \quad \rho(t)=1$ if $t<1-2 \varepsilon, \quad \rho(t)=0 \quad$ if $t>1-\varepsilon, \quad \rho^{\prime}(t) \leqq 0$, $\varphi \in C_{0}^{\infty}\left(\mathbf{R}^{n}-\{0\}\right), \varphi \geqq 0, \chi(x) \geqq 0, \chi(x)=1$ if $|x|>2, \chi(x)=0$ if $|x|<1$.

Lemma 4.1. Let $m>-1 / 2$ and $p(x, \xi) \in S_{-}^{2 m}$ be a symbol of canonical type. Let $a(x, \xi)=(|x||\xi|-x \cdot \xi) p(x, \xi)$. Then there exists a constant $C_{0}>0$ such that

$$
-\left\{|\xi|^{2}, a\right\} \geqq C_{0} p+q,
$$

where $\{$,$\} denotes the Poisson bracket and q$ is compactly supported in $x$.

Proof. Modulo a function of compact support in $x$,

$$
\begin{aligned}
-\left\{|\xi|^{2}, a\right\}= & 2(2 m+1)|\xi|^{2}(1-\hat{x} \cdot \hat{\xi}) p(x, \xi) \\
& -2|\xi|(|x||\xi|-x \cdot \xi)^{2 m+1}|x|^{-1}\left(1-(\hat{x} \cdot \hat{\xi})^{2}\right) \rho^{\prime}(\hat{x} \cdot \hat{\xi}) \varphi(\xi) \chi(x) .
\end{aligned}
$$

The second term is nonnegative since $\rho^{\prime}(t) \leqq 0$. On the support of $p(x, \xi)$, $2(2 m+1)|\xi|^{2}(1-\hat{x} \cdot \hat{\xi})$ is estimated from below by a constant $C_{0}>0$.

With a symbol $p(x, \xi)$, we associate the Weyl quantization $P=p^{W}\left(x, D_{x}\right)$ (see e.g. [9]).

Lemma 4.2. Let $p(x, \xi) \in S_{-}^{m}$ be a real symbol. Let $Q$ be the Weyl quantization of $p(x, \xi)^{2}$. Then we have $-Q \leqq P_{1}+P_{N}$ in the form sense, where $P_{1} \in S_{-}^{2 m-2}$, $P_{N} \in S^{-N}, N$ being a sufficiently large constant.

Proof. Let $P=p^{W}\left(x, D_{x}\right)$. Then $P^{2}=Q+P_{1}+P_{N}$, with $P_{1} \in S_{-}^{2 m-2}, P_{N} \in S^{-N}$. $P^{2} \geqq 0$, since $P$ is symmetric.

Let $H=-\Delta+V$ be the Schrödinger operator on $\mathbf{R}^{n}$ where $V$ is a real function satisfying

$$
\partial_{x}^{\alpha} V=O\left(\langle x\rangle^{-|\alpha|-\rho}\right), \quad 0<\rho<1
$$


Let $p$ and $a$ be as in Lemma 4.1 and set $A=a^{W}\left(x, D_{x}\right), P=p^{W}\left(x, D_{x}\right)$. Let $C_{0} p+q$ be as in Lemma 4.1. One can construct $p(x, \xi)$ in such a way that the square root of $-\left\{|\xi|^{2}, a\right\}-\left(C_{0} p+q\right)$ is $C^{\infty}$. So, by Lemmas 4.1 and 4.2, we have

Lemma 4.3. $C_{0} P \leqq-i[H, A]+P_{1}+P_{N}$ in the form sense, where $P_{1} \in S_{-}^{2 m-\rho}$, $P_{N} \in S^{-N}, N$ being a sufficiently large constant.

Let $u=(H-z)^{-1} f, \operatorname{Im} z>0$. Then by Lemma 4.3 we have

$$
C_{0}(P u, u) \leqq-i([H, A] u, u)+\left(P_{1} u, u\right)+\left(P_{N} u, u\right) .
$$

The first term of the right-hand side is calculated as

$$
-i([H, A] u, u)=-2 \operatorname{Im} z(A u, u)-i\{(A u, f)-(f, A u)\} .
$$

One can assume that the square root of $a(x, \xi)$ is $C^{\infty}$. So, by Lemma 4.2, the first term of the right-hand side of (4.2) is dominated by

$$
\left(P_{1} u, u\right)+\left(P_{N} u, u\right), \quad P_{1} \in S_{-}^{2 m-1}, \quad P_{N} \in S^{-N} .
$$

We also have

$$
\begin{aligned}
|(A u, f)| & \leqq \frac{1}{2}\left(\|A u\|_{-m-1-\rho}^{2}+\|f\|_{m+1+\rho}^{2}\right) \\
& \leqq\left(P_{1} u, u\right)+C\|f\|_{m+1+\rho}^{2}, \quad P_{1} \in S_{-}^{2 m-2 \rho} .
\end{aligned}
$$

These estimates together with (4.1) show that

$$
(P u, u) \leqq\left(P_{1} u, u\right)+C\|f\|_{m+1+\rho}^{2}, \quad P_{1} \in S_{-}^{2 m-\rho} .
$$

Here we note that by enlarging the support suitably, we can dominate the symbol of $P_{1} \in S_{-}^{2 m-\rho}$ from above by the symbol of canonical type $\in S_{-}^{2 m-\rho}$. So, one can use (4.5) with $2 m$ replaced by $2 m-\rho$ to estimate $\left(P_{1} u, u\right)$. We repeat this procedure and finally obtain

$$
(P u, u) \leqq C\|f\|_{m+1+\rho}^{2},
$$

which implies that

$$
\|P u\| \leqq C\|f\|_{m+1+\rho},
$$

if $P \in S_{-}^{m}, m>-1 / 2$.

Now we turn to the three body problem and prove Theorem 3.6. Let $S_{-}^{m}$ be as above with $x, \xi$ replaced by $x_{\beta}, \xi_{\beta}$. We introduce $P$ and $A$ in the same way as in Lemma 4.1 with $x$, $\xi$ replaced by $x_{\beta}, \xi_{\beta}$. Let $u=R(z) f, z=\lambda+i \varepsilon$.

We first note that

$$
-i\left[H, \chi_{\beta} A \chi_{\beta}\right]=-i \chi_{\beta}[H, A] \chi_{\beta}-i\left[H, \chi_{\beta}\right] A \chi_{\beta}-i \chi_{\beta} A\left[H, \chi_{\beta}\right],
$$

and that

$$
-i[H, A]=-i\left[-\Delta_{x_{\beta}}, A\right]-i \sum_{\gamma \neq \beta}\left[V_{\gamma}, A\right] .
$$


To the term $-i\left[-\Delta_{x_{\beta}}, A\right]$, we apply Lemma 4.1. On the support of $\chi_{\beta}(x), V_{\gamma}\left(x^{\gamma}\right)$ $(\gamma \neq \beta)$ behaves like $\left\langle x_{\beta}\right\rangle^{-\rho}, \rho>0$. Therefore arguing as in the proof of Lemma 4.3 , we have

$$
\begin{aligned}
C_{0}\left(\chi_{\beta} P \chi_{\beta} u, u\right) \leqq & -i\left(\chi_{\beta}[H, A] \chi_{\beta} u, u\right)+\sum_{j}^{\text {finite }}\left(\tilde{\chi}_{\beta}^{(j)} P_{1}^{(j)} \tilde{\chi}_{\beta}^{(j)} u, u\right) \\
& +C_{N}\left\|\langle x\rangle^{-N} u\right\|^{2},
\end{aligned}
$$

where $P_{1}^{(j)} \in S_{-}^{2 m-\rho}$ and $\tilde{\chi}_{\beta}^{(j)}(x)$ is a cut off function similar to $\chi_{\beta}(x)$ and $N$ is a sufficiently large constant.

We claim that

$$
\left|\left(\left[H, \chi_{\beta}\right] A \chi_{\beta} u, u\right)\right| \leqq C\|f\|_{m+1+\rho}^{2} .
$$

Let $\varphi(\cdot)$ be a smooth cut off function near $\lambda$. Then as is well-known, for any $s \in \mathbf{R}$,

$$
\langle x\rangle^{s}(1-\varphi(H)) R(\lambda \pm i \varepsilon)\langle x\rangle^{-s} \in \mathbf{B}\left(L^{2}(X) ; L^{2}(X)\right),
$$

uniformly in $\varepsilon>0$. On the other hand, by [2] Lemma 2.1 , we have

$$
\begin{aligned}
& \varphi(H)\left[H, \chi_{\beta}\right] A \chi_{\beta} \\
= & \varphi\left(H_{0}\right)\left[H, \chi_{\beta}\right] A \chi_{\beta}+\sum_{k}^{\text {finite }}\langle x\rangle^{-\rho k} p_{k}\left(x, D_{x}\right) \varphi_{k}\left(H_{0}\right)\left[H, \chi_{\beta}\right] A \chi_{\beta}+R_{N},
\end{aligned}
$$

where $p_{k}(x, \xi)$ satisfies (2.1) and (2.2) of [2], supp $\varphi_{k} \subset \operatorname{supp} \varphi$ and $\langle x\rangle^{N / 2} R_{N}\langle x\rangle^{N / 2}$ $\in \mathbf{B}\left(L^{2}(X) ; L^{2}(X)\right)$. Now we note that on the support of the symbol of $\varphi\left(H_{0}\right)\left[H, \chi_{\beta}\right] A \chi_{\beta}, x \cdot \xi \leqq \mu|x||\xi|,-1<\mu<1$, which follows from the fact that $x_{\beta} \cdot \xi_{\beta}<(1-\varepsilon)\left|x_{\beta}\right|\left|\xi_{\beta}\right|$. One can then apply [2], Theorem 2.2 to estimate this term. The terms $\langle x\rangle^{-\rho k} p_{k}\left(x, D_{x}\right) \varphi_{k}\left(H_{0}\right)\left[H, \chi_{\beta}\right] A \chi_{\beta}$ are treated similarly. This proves (4.9).

In view of $(4.6) \sim(4.9)$ and arguing similarly to the 2-body case, we have

$$
\left(\chi_{\beta} P \chi_{\beta} u, u\right) \leqq \sum_{j}^{\text {finite }}\left(\tilde{\chi}_{\beta}^{(j)} P_{1}^{(j)} \tilde{\chi}_{\beta}^{(j)} u, u\right)+C\|f\|_{m+1+\rho}^{2},
$$

where $P_{1}^{(j)} \in S_{-}^{2 m-\rho}$ and $\tilde{\chi}_{\beta}^{(j)}(x)$ is cut off function similar to $\chi_{\beta}(x)$. The rest of the proof is the same as in the 2 -body case.

\section{Proof of Theorem 1.3}

For a small $\varepsilon>0$ we take $\psi_{\beta}(t) \in C^{\infty}\left(\mathbf{R}^{1}\right)$ such that $\psi_{\beta}(t)=1$ if $t>\lambda-\varepsilon, \psi_{\beta}(t)=0$ if $t<\lambda-2 \varepsilon$. Let $\psi_{\beta}\left(D_{x_{\beta}}\right)$ be the Ps.D.Op. with symbol $\psi_{\beta}\left(\left|\xi_{\beta}\right|^{2}\right)$ and put $w=\psi_{\beta}\left(D_{x_{\beta}}\right) \chi_{\beta}(x) v$, where $v$ is defined by $(1.11)$ and $\chi_{\beta}(x)$ is given in Sect. 3. Note that by our assumption $f$ defined by (1.12) is rapidly decreasing. A simple calculation shows that for any $N>0$,

$$
\chi_{\beta}(x) \psi_{\beta}\left(D_{x_{\beta}}\right) v=\psi_{\beta}\left(D_{x_{\beta}}\right) \chi_{\beta}(x) v+O\left(\left|x_{\beta}\right|^{-N}\right),
$$

as $\left|x_{\beta}\right| \rightarrow \infty$ uniformly for $\left|x^{\beta}\right|<a$. So, we have only to consider the asymptotic behavior of $w$. 
Let $g=\left(H_{\beta}-\lambda\right) w$. Then by the same arguments as in the proof of [2], Lemma 4.2 , we have $g \in L^{2, s}$ for all $s \geqq 0$. Hence

$$
w=R_{\beta}(\lambda+i 0) g .
$$

Let $P_{p}\left(h^{\beta}\right)$ denote the projection onto the point spectrum of $h^{\beta}$. Then

$$
\left(P_{p}\left(h^{\beta}\right) \otimes 1\right) w=\sum_{l} P_{l}^{\beta} \otimes\left(-\Delta_{x_{\beta}}-\left(\lambda-E_{l}^{\beta}+i 0\right)\right)^{-1} g,
$$

where $P_{l}^{\beta}$ denotes the projection onto the eigenspace of $h^{\beta}$ with eigenvalue $E_{l}^{\beta}$.

Lemma 5.1. Let $r=\left|x_{\beta}\right|, \theta_{\beta}=x_{\beta} / r$. Then we have the following expansion:

$$
\begin{aligned}
P_{l}^{\beta} \otimes\left(-\Delta_{x_{\beta}}-\left(\lambda-E_{l}^{\beta}+i 0\right)\right)^{-1} g & \simeq C_{\beta l}(\lambda) r^{-1} e^{i \sqrt{\lambda-E_{l}^{\beta}} A_{\beta l}\left(\lambda ; \theta_{\beta}, \omega\right) u_{\beta l}\left(x^{\beta}\right),} \\
C_{\beta l}(\lambda) & =2 \pi i\left(\lambda-E^{\alpha}\right)^{-1 / 4}\left(\lambda-E_{l}^{\beta}\right)^{-1 / 4},
\end{aligned}
$$

as $r \rightarrow \infty$ uniformly for $\theta_{\beta} \in S^{2}$.

Proof. We proceed in the same way as in [2], $\S 5$. We define $\mathscr{F}_{\beta l}(\lambda)$ in the same way as in (1.7) with $E^{\alpha}$ replaced by $E_{l}^{\beta}$. Let $\chi_{\beta}$ and $\psi_{\beta}$ be as above and define $P$ and $G$ by

$$
P=\chi_{\beta}(x) \psi_{\beta}\left(D_{x_{\beta}}\right), \quad G=H P-P H_{\beta} .
$$

Then by the same argument as in the proof of [2], Lemma 5.2, we see that $A_{\beta l}\left(\lambda ; \theta_{\beta}, \omega\right)$ is represented by

$$
A_{\beta l}\left(\lambda ; \theta_{\beta}, \omega\right)=-2 \pi i C_{\alpha}(\lambda) \mathscr{F}_{\beta l}(\lambda) J_{\beta l}^{*}\left(P^{*} f-G^{*} R(\lambda+i 0) f\right),
$$

where $J_{\beta l}$ is the injection defined in the same way as in (1.4) with $u_{\alpha}$ replaced by $u_{\beta l}$ and $f$ is defined by (1.12). Let $v=R(\lambda+i 0) f$. Then

$$
P^{*} f-G^{*} R(\lambda+i 0) f=\left(H_{\beta}-\lambda\right) P^{*} v .
$$

Since $w=P^{*} v$, the right-hand side is equal to $g$. So, we have

$$
A_{\beta l}\left(\lambda ; \theta_{\beta}, w\right)=-2 \pi i C_{\alpha}(\lambda) \mathscr{F}_{\beta l}(\lambda) J_{\beta l}^{*} g .
$$

Let $\tilde{w}$ be defined by

$$
\begin{aligned}
\tilde{w} & =\left(-\Delta_{x_{\beta}}-\left(\lambda-E_{l}^{\beta}+i 0\right)\right)^{-1} J_{\beta l}^{*} g, \\
J_{\beta l}^{*} g\left(x_{\beta}\right) & =\int_{\mathbf{R}^{3}} \overline{u_{\beta l}\left(x^{\beta}\right)} g(x) d x^{\beta} .
\end{aligned}
$$

Since $J_{\beta l}^{*} g$ is rapidly decreasing, we have by Lemma 2.1 ,

$$
\begin{aligned}
\tilde{w} & \simeq C(\lambda) r^{-1} e^{i \sqrt{\lambda-E_{l}^{\beta}} r} \mathscr{F}_{\beta l}(\lambda) J_{\beta l}^{*} g, \\
C(\lambda) & =\pi^{1 / 2}\left(\lambda-E_{l}^{\beta}\right)^{-1 / 4},
\end{aligned}
$$

as $r=\left|x_{\beta}\right| \rightarrow \infty$ uniformly for $\theta_{\beta} \in S^{2}$. Equations (5.3) and (5.4) imply that

$$
\begin{aligned}
\tilde{w} & \simeq C_{\beta l}(\lambda) r^{-1} e^{i \sqrt{\lambda-E_{l}^{\beta}} r} A_{\beta l}\left(\lambda ; \theta_{\beta}, \omega\right), \\
C_{\beta l}(\lambda) & =2 \pi i\left(\lambda-E^{\alpha}\right)^{-1 / 4}\left(\lambda-E_{l}^{\beta}\right)^{-1 / 4} .
\end{aligned}
$$

Lemma 5.1 immediately follows from (5.5). 
Let $P_{\mathrm{ac}}\left(h^{\beta}\right)$ denote the projection onto the absolutely continuous subspace for $h^{\beta}$. It remains to show that the asymptotic behavior of $\left(P_{\mathrm{ac}}\left(h^{\beta}\right) \otimes 1\right) w$ is negligible compared with that of $\left(P_{p}\left(h^{\beta}\right) \otimes 1\right) w$. For that purpose, we recall some results on the 2-body Schrödinger operators.

Let $H_{0}=-\Delta, H=-\Delta+V$ be the Schrödinger operators in $\mathbf{R}^{3}$. Suppose that $V(x)$ is a rapidly decreasing function. Let $R(z)=(H-z)^{-1}$. We define the usual Fourier transformation $T_{0}$ and the generalized Fourier transformation $T$ by

$$
\begin{aligned}
\left(T_{0} f\right)(\xi) & =(2 \pi)^{-3 / 2} \int_{\mathbf{R}^{3}} e^{-i x \cdot \xi} f(x) d x, \\
(T f)(\xi) & =(2 \pi)^{-3 / 2} \int_{\mathbf{R}^{3}} e^{-i x \cdot \xi}\left(f(x)-V(x) R\left(|\xi|^{2}+i 0\right) f\right) d x .
\end{aligned}
$$

Then as is well-known, $T$ is a partial isometry with initial set $E((0, \infty)) L^{2}\left(\mathbf{R}^{3}\right)$ and final set $L^{2}\left(\mathbf{R}^{3}\right)$, where $E(\lambda)$ denotes the spectral measure for $H$. $E((0, \infty)) L^{2}\left(\mathbf{R}^{3}\right)$ coincides with the absolutely continuous subspace for $H$. Let $\Omega=T^{*} T_{0}$ be the stationary wave operator, which is known to be equal to the time-dependent wave operator.

Lemma 5.2. Let $\varphi(t) \in C^{\infty}\left(\mathbf{R}^{1}\right)$ be such that for some $a>0, \varphi(t)=0$ if $t<a$ and $\left|\varphi^{(m)}(t)\right| \leqq C_{m} t^{-1-m / 2}, m=0,1,2, \ldots$, for any $t$. Then for any $s^{\prime}>s \geqq 0$,

$$
\langle x\rangle^{-s^{\prime}} \varphi(H) \Omega\langle x\rangle^{s} \in \mathbf{B}\left(L^{2}\left(\mathbf{R}^{3}\right) ; L^{2}\left(\mathbf{R}^{3}\right)\right) \text {. }
$$

Proof. Let $H^{m}$ denote the Sobolev space of order $m$. Then by [3], Theorem 0.1, $T \varphi(H)\langle x\rangle^{-N-1}$ is bounded from $L^{2}\left(\mathbf{R}_{x}^{3}\right)$ to $H^{N}\left(\mathbf{R}_{\xi}^{3}\right)$, which shows that $\langle x\rangle^{N} T_{0}^{*} T \varphi(H)\langle x\rangle^{-N-1}=\langle x\rangle^{N} \Omega^{*} \varphi(H)\langle x\rangle^{-N-1}$ is bounded in $L^{2}\left(\mathbf{R}^{3}\right)$. The lemma then follows from the interpolation.

For $\lambda>0$, we set $(T(\lambda) f)(\omega)=2^{-1 / 2} \lambda^{1 / 4}(T f)(\sqrt{\lambda} \omega)$, which is well-defined when $f \in L^{2, s}, s>1 / 2$. Then for $f, g \in L^{2, s}(s>1 / 2)$ and $0<a<b<\infty$, we have

$$
\int_{a}^{b}(T(\lambda) f, T(\lambda) g)_{L^{2}\left(S^{2}\right)} d \lambda=(E((a, b)) f, g) .
$$

Lemma 5.3. If $s>5 / 2$, there exists a constant $C>0$ such that

$$
\left\|T(\lambda)^{*} T(\lambda)\right\|_{\mathbf{B}\left(L^{2, s} ; L^{2,-s}\right)} \leqq C / \sqrt{\lambda}, \quad \text { if } 0<\lambda<1 .
$$

Proof. Equation (5.8) shows that

$$
T(\lambda)^{*} T(\lambda)=\frac{1}{2 \pi i}(R(\lambda+i 0)-R(\lambda-i 0)) .
$$

The lemma then follows from [4].

We now turn to our problem. Let $h_{0}^{\beta}=-\Delta_{x^{\beta}}, h^{\beta}=h_{0}^{\beta}+V_{\beta}\left(x^{\beta}\right)$. Let $T_{0}$ and $T_{\beta}$ be defined by (5.6) and (5.7) with $H_{0}$ and $H$ replaced by $h_{0}^{\beta}$ and $h^{\beta}$, respectively. Let $\Omega^{\beta}=\left(T_{\beta}\right) * T_{0}$. We choose $\varphi_{0}(t), \varphi_{1}(t) \in C^{\infty}\left(\mathbf{R}^{1}\right)$ such that $\varphi_{0}(t)+\varphi_{1}(t)=1$, $\varphi_{0}(t)=1$ if $t<1 / 2, \varphi_{1}(t)=1$ if $t>2$. We define the operators $F\left(h^{\beta} \leqq \varepsilon\right)$ and $F\left(h^{\beta} \geqq \varepsilon\right)$ by $F\left(h^{\beta} \leqq \varepsilon\right)=\varphi_{0}\left(h^{\beta} / \varepsilon\right), F\left(h^{\beta} \geqq \varepsilon\right)=\varphi_{1}\left(h^{\beta} / \varepsilon\right)$. In the sequel we omit the symbol $\otimes$. We split $P_{\text {ac }}\left(h^{\beta}\right) w$ into two parts:

$$
P_{\mathrm{ac}}\left(h^{\beta}\right) w=P_{\mathrm{ac}}\left(h^{\beta}\right) F\left(h^{\beta} \leqq \varepsilon\right) w+F\left(h^{\beta} \geqq \varepsilon\right) w .
$$


We first note that by the intertwining property $R_{\beta}(z) P_{\mathrm{ac}}\left(h^{\beta}\right)=\Omega^{\beta} R_{0}(z)\left(\Omega^{\beta}\right)^{*}$, where $R_{0}(z)$ is the resolvent of $-\Delta$ in $\mathbf{R}^{6}$. Then, we have

$$
F\left(h^{\beta} \geqq \varepsilon\right) w=F\left(h^{\beta} \geqq \varepsilon\right) \Omega^{\beta} R_{0}(\lambda+i 0)\left(\Omega^{\beta}\right)^{*} \tilde{F}\left(h^{\beta} \geqq \varepsilon / 4\right) g,
$$

where $\tilde{F}\left(h^{\beta} \geqq \varepsilon / 4\right)$ is an operator similar to $F\left(h^{\beta} \geqq \varepsilon\right)$ which cuts off the part $h^{\beta} \leqq \varepsilon / 4$. By the Sobolev inequality, we have

$$
\begin{aligned}
& \left\|F\left(h^{\beta} \geqq \varepsilon\right) \Omega^{\beta}\left(h_{0}^{\beta}+1\right)^{-2}\left\langle x^{\beta}\right\rangle^{s}\right\|_{\mathbf{B}\left(L^{2}\left(\mathbf{R}^{3}\right) ; L^{\alpha}\left(\left|x^{\beta}\right|<a\right)\right)} \\
& \leqq \\
& \quad C\left(\left\|F\left(h^{\beta} \geqq \varepsilon\right) h^{\beta} \Omega^{\beta}\left(h_{0}^{\beta}+1\right)^{-2}\left\langle x^{\beta}\right\rangle^{s}\right\|_{\mathbf{B}\left(L^{2}\left(\mathbf{R}^{3}\right) ; L^{2}\left(\left|x^{\beta}\right|<a+1\right)\right)}\right. \\
& \quad+\left\|F\left(h^{\beta} \geqq \varepsilon\right) \Omega^{\beta}\left(h_{0}^{\beta}+1\right)^{-2}\left\langle x^{\beta}\right\rangle^{s}\right\|_{\mathbf{B}\left(L^{2}\left(\mathbf{R}^{3}\right) ; L^{2}\left(\left|x^{\beta}\right|<a+1\right)\right)},
\end{aligned}
$$

the last term being finite for any $s>0$ by Lemma 5.2. Therefore,

$$
\begin{gathered}
\|\left(F\left(h^{\beta} \geqq \varepsilon\right) w\left(x^{\beta}, x_{\beta}\right) \|_{L^{\infty}\left(\left|x^{\beta}\right|<a\right)}\right. \\
\leqq C\left\|\left\langle x^{\beta}\right\rangle^{-s}\left(R_{0}(\lambda+i 0)\left(1+h_{0}^{\beta}\right)^{2}\left(\Omega^{\beta}\right)^{*} \widetilde{F}\left(h^{\beta} \geqq \varepsilon / 4\right) g\right)\left(x^{\beta}, x_{\beta}\right)\right\|_{L^{2}\left(\mathbf{R}_{x^{\prime}}^{3}\right)} .
\end{gathered}
$$

Let $\tilde{g}=\left(1+h_{0}^{\beta}\right)^{2}\left(\Omega^{\beta}\right)^{*} \tilde{F}\left(h^{\beta} \geqq \varepsilon / 4\right) g$. Since $g$ is rapidly decreasing, so is $\tilde{g}$ by virtue of Lemma 5.2. We then have

$$
\left|\left(R_{0}(\lambda+i 0) \tilde{g}\right)(x)\right| \leqq C\left(1+\left|x^{\beta}\right|+\left|x_{\beta}\right|\right)^{-5 / 2},
$$

by the well-known property of the Hankel function. Hence

$$
\left\|\left(F\left(h^{\beta} \geqq \varepsilon\right) w\right)\left(x^{\beta}, x_{\beta}\right)\right\|_{L^{\infty}\left(\left|x^{\beta}\right|<a\right)} \leqq C\left(1+\left|x_{\beta}\right|\right)^{-5 / 2} .
$$

We next consider $P_{\text {ac }}\left(h^{\beta}\right) F\left(h^{\beta} \leqq \varepsilon\right) w$. Passing to the generalized Fourier transform, we have

$$
P_{\mathrm{ac}}\left(h^{\beta}\right) F\left(h^{\beta} \leqq \varepsilon\right) w=\int_{0}^{2 \varepsilon} \varphi_{0}(k / \varepsilon)\left(-\Delta_{x_{\beta}}-\lambda+k-i 0\right)^{-1} T_{\beta}(k)^{*} T_{\beta}(k) g d k .
$$

By the Sobolev inequality, we have

$$
\begin{aligned}
& \left\|\left(P_{\mathrm{ac}}\left(h^{\beta}\right) F\left(h^{\beta} \leqq \varepsilon\right) w\right)\left(x^{\beta}, x_{\beta}\right)\right\|_{L^{\infty}\left(\left|x^{\beta}\right|<a\right)} \\
& \leqq C\left(\left\|\left(P_{\mathrm{ac}}\left(h^{\beta}\right) h^{\beta} F\left(h^{\beta} \leqq \varepsilon\right) w\right)\left(x^{\beta}, x_{\beta}\right)\right\|_{L^{2}\left(\left|x^{\beta}\right|<a+1\right)}\right. \\
& \left.\quad+\left\|\left(P_{\mathrm{ac}}\left(h^{\beta}\right) F\left(h^{\beta} \leqq \varepsilon\right) w\right)\left(x^{\beta}, x_{\beta}\right)\right\|_{L^{2}\left(\left|x^{\beta}\right|<a+1\right)}\right) \\
& \leqq C \int_{0}^{2 \varepsilon}\left\|\left(\left(-\Delta_{x_{\beta}}-\lambda+k-i 0\right)^{-1} T_{\beta}(k)^{*} T_{\beta}(k) g\right)\left(x^{\beta}, x_{\beta}\right)\right\|_{L^{2}\left(\left|x^{\beta}\right|<a+1\right)} d k,
\end{aligned}
$$

$C$ being independent of $\varepsilon$ and $x_{\beta}$. Using the decay property of the Green's function of $-\Delta_{x_{\beta}}$, we have

$$
\begin{aligned}
& \left|\left(\left(-\Delta_{x_{\beta}}-\lambda+k-i 0\right)^{-1} T_{\beta}(k)^{*} T_{\beta}(k) g\right)\left(x^{\beta}, x_{\beta}\right)\right| \\
\leqq & C\left(1+\left|x_{\beta}\right|\right)^{-1}\left\|\left\langle x_{\beta}\right\rangle^{s}\left(T_{\beta}(k)^{*} T_{\beta}(k) g\right)\left(x^{\beta}, x_{\beta}\right)\right\|_{L^{2}\left(\mathbf{R}_{x_{s}}^{3}\right)},
\end{aligned}
$$

for sufficiently large $s>0$. We have, therefore,

$$
\begin{gathered}
\left\|\left(P_{\mathrm{ac}}\left(h^{\beta}\right) F\left(h^{\beta} \leqq \varepsilon\right) w\right)\left(x^{\beta}, x_{\beta}\right)\right\|_{L^{\infty}\left(\left|x^{\beta}\right|<a\right)} \\
\leqq C\left(1+\left|x_{\beta}\right|\right)^{-1} \int_{0}^{2 \varepsilon}\left\|\left\langle x_{\beta}\right\rangle^{s}\left\langle x^{\beta}\right\rangle^{-s} T_{\beta}(k)^{*} T_{\beta}(k) g\right\|_{L^{2}\left(\mathbf{R}^{6}\right)} d k .
\end{gathered}
$$


Since $g$ is rapidly decreasing, by Lemma 5.3, the above integral is dominated by

$$
\left(1+\left|x_{\beta}\right|\right)^{-1} \int_{0}^{2 \varepsilon} k^{-1 / 2} d k=C\left(1+\left|x_{\beta}\right|\right)^{-1} \sqrt{\varepsilon} .
$$

Equations (5.9) and (5.10) show that if $\left|x^{\beta}\right|<a, P_{\mathrm{ac}}\left(h^{\beta}\right) w=o\left(r^{-1}\right)$ as $r=\left|x_{\beta}\right|$ $\rightarrow \infty$, which completes the proof of Theorem 1.3 .

Acknowledgement. The author deeply thanks Professor V. Buslaev who indicated to him many physical references. He also thanks Professors J. Derezinski, C. Gérard, K.B. Sinha and E. Skibsted for their helpful discussions.

\section{References}

1. Gérard, C.: Sharp propagation estimates for N-particle systems. Duke Mach. J. 67, 483-515 (1992)

2. Isozaki, H.: Structures of S-matrices for three body Schrödinger operators. Commun. Math. Phys. 146, 241-258 (1992)

3. Isozaki, H.: Differentiability of generalized Fourier transforms associated with Schrödinger operators. J. Math. Kyoto Univ. 25, 789-806 (1985)

4. Jensen, A. and Kato, T.: Spectral properties of Schrödinger operators and time decay of the wave functions. Duke Math. J. 46, 583-611 (1979)

5. Mercuriev, S.P.: On the three-body Coulomb scattering problem. Ann. Phys. 130, 395-426 (1980)

6. Newton, R.G.: The asymptotic form of the three-particle wave functions and the cross sections. Ann. Phys. 74, 324-351 (1972)

7. Newton, R.G.: The three particle S-matrix. J. Math. Phys. 15, 338-343 (1974)

8. Nuttal, J.: Asymptotic form of the three-particle scattering wave functions for free incident particles. J. Math. Phys. 12, 1896-1899 (1971)

9. Robert, D.: Autour de l'approximation semiclassique. Basel-Boston: Birkhäuser 1983

10. Saito, Y.: Spectral representation for Schrödinger operators with long-range potentials. Lect. Notes in Math. 727, Berlin, Heidelberg, New York: Springer 1979

11. Sigal, I.M. and Soffer, A.: Local decay and propagation estimates for time-dependent and time-independent Hamiltonians. Preprint 1988

12. Skibsted, E.: Propagation estimates for N-body Schrödinger operators. Commun. Math. Phys. 142, 67-98 (1991)

Communicated by H. Araki 
